\title{
RESEARCH PERSPECTIVES \\ Characterizing Brain Tumor Research: The Role of the National Institutes of Health
}

W.B. Pope

M.W. Itagaki
SUMMARY: The contribution of radiology to brain tumor research is unknown. We sought to determine how the proportion of neuro-oncologic publications generated by radiology departments has changed and if there is an association with NIH funding levels. Therefore we searched The National Library of Medicine's PubMed database for all articles published on brain neoplasms from 1996 to 2007. Country and department of origin and $\mathrm{NIH}$ grant support were noted for each article. Approximately $10 \%$ of brain tumor publications originated from radiology departments, ranking third among medical specialties. NIH funding for this research grew from less than $20 \%$ in 1996 to more than $50 \%$ in 2007 . Overall $\mathrm{NIH}$ funding levels rose approximately 2.5 fold during this time. The U.S. was the dominant producer of brain tumor publications, and the gap between the U.S. and the rest of the world grew over the study period. Thus a substantial proportion of brain tumor publications originate from radiology departments, and the percentage of this research that is funded by the NIH has grown significantly during a period of increasing $\mathrm{NIH}$ budgets.

ABBREVIATIONS: AJNR = American Journal of Neuroradiology; CNS = central nervous system; $\mathrm{GBM}=$ glioblastoma multiforme; Heme-Onc = hematology-oncology; $\mathrm{MeSH}=$ medical subject headings; $\mathrm{NCl}=$ National Cancer Institute; $\mathrm{NIH}=$ National Institutes of Health
I $\mathrm{n}$ the United States, it is estimated that approximately 22000 people will be diagnosed with a primary malignant CNS tumor in 2009, and approximately 12900 people will die from their disease. ${ }^{1}$ Brain tumors are the third leading cause of death from cancer in individuals from 20 to 39 years of age. Survival rates for GBM, the most common and deadly primary brain tumor in adults, remain low, at no more than $3.3 \%$ at 5 years, and overall brain cancer mortality rates have changed little during the past 20 years. ${ }^{2,3}$ Thus, it is likely that substantial further investment in brain tumor research will be required to have a meaningful impact on morbidity and mortality in GBM and other neuro-oncologic diseases.

The level of NIH funding is the single greatest predictor of research output among US radiology departments. ${ }^{4}$ Funding and publications are strongly correlated. In the United States, approximately $86 \%$ of the number of radiology department publications can be predicted by NIH funding levels alone. ${ }^{3}$ For US medical schools, there is a correlation coefficient of 0.95 between NIH funding levels and the quantity of publications. ${ }^{5}$ Radiology departments with the highest funding levels receive $70 \%$ of research financing from the $\mathrm{NIH},{ }^{6}$ and in 2003 , this accounted for approximately $3.3 \%$ of grant funds awarded to the associated medical schools. ${ }^{7}$ These data suggest a positive effect of federal funding on biomedical research.

While recently stagnant, NIH funding levels are scheduled to get a one-time boost of $\$ 8.2$ billion for 2 years in extramural funding associated with the American Recovery and Reinvestment Act of 2009 (http://grants.nih.gov/recovery). ${ }^{8,9} \mathrm{NIH}$

Received July 2, 2009; accepted after revision August 31

From the Department of Radiological Sciences, David Geffen School of Medicine at the University of California Los Angeles, Los Angeles, California.

Paper previously presented at: Annual Meeting of the American Society of Neuroradiology; May 16-21, 2009; Vancouver, British Columbia, Canada.

Please address correspondence to Whitney B. Pope, MD, PhD, Department of Radiological Sciences, David Geffen School of Medicine at the University of California, Los Angeles, 10833 Le Conte Ave, BL-428 CHS, Los Angeles, CA 90095-1721; e-mail: wpope@ mednet.ucla.edu

DOI 10.3174/ajnr.A1904 funding for brain tumor research will grow to $\$ 200$ million in 2009 (projected; NCI Office of Budget and Finance. http:// obf.cancer.gov/). Despite this tremendous research investment in the United States and worldwide, there is little analysis as to the publication output, funding levels, and contributions of various medical specialties and countries to this process and whether these variables are static or changing significantly. Thus, we categorized neuro-oncologic publications by medical specialty and country of origin and assessed correlation with NIH funding levels during a recent 12-year period.

\section{Materials and Methods}

Article Search. This was a retrospective analysis of a publically available data base of research publications and was exempt from institutional review board approval. The PubMed data base of the National Library of Medicine was searched for all articles with the primary MeSH keywords "brain neoplasms" that were published from 1996 to 2007 (12 years), encompassing all years for which complete data, including impact factor, were available in electronic form. "Brain neoplasms" is the term used by MEDLINE to denote articles about brain tumors of all kinds. Country, department of origin, and NIH grant support, if present, were recorded for each article. Department of origin corresponds to the department of the first author. Journal impact factors were obtained from Thompson Reuters for the journal in which the article was published in the year of publication (Thompson Reuters. Journal Citation Reports. http://scientific. thomson.com/products/jcr/. Accessed October 11, 2008).

Data Collection. The matching citation data were downloaded and reconstructed in a local data base as previously described. ${ }^{10}$ Briefly, the descriptor fields for each article, including the article publication date, author affiliation, and article methodology, were recorded. Only articles that were flagged as journal articles, the PubMed designation for a report in a journal, were included. Editorials, comments, letters, news items, bibliographies, and other nonscientific content are typically not indexed as journal articles and were thus excluded. Methodologic and article type categories, as specified by MEDLINE, were retained. These included the following: case reports; review articles; meta-analyses; multicenter studies; general clinical 


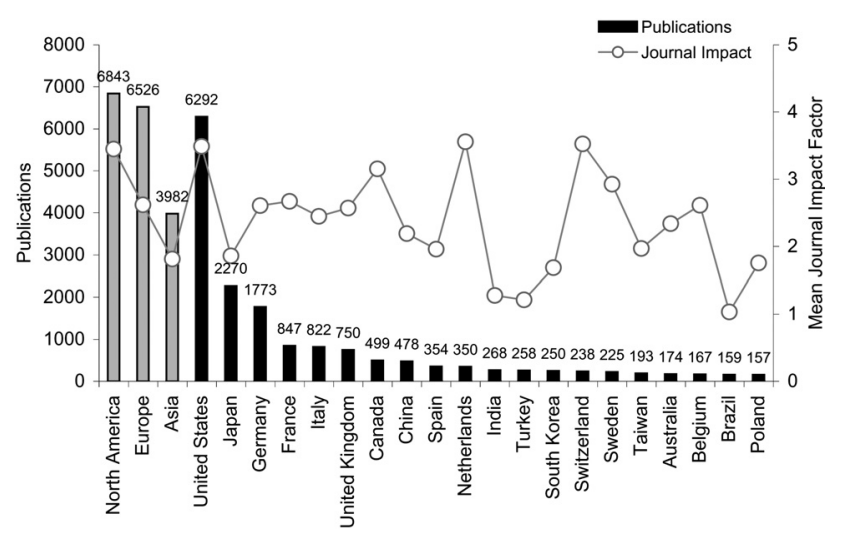

Fig 1. Total published brain tumor articles by country/region during the 12-year study period (1996-2007), with mean journal impact factor.

trials; and controlled, randomized-controlled, phase I, phase II, phase III, and phase IV clinical trials. Additionally, a category of "multicenter trial" was created for articles that were both clinical trials and multicenter studies, and a category of "general research article" was created for articles that were classified as journal articles but belonged to no other article type.

Further details on PubMed methodology types can be found at the National Library of Medicine Website (http://www.nlm.nih. gov/mesh/pubtypes2008.html). If the article received NIH funding, the NIH institute and grant number, indexed fields in the PubMed citation, were recorded. The first author's affiliation field contains the department, institution, city, state (if applicable), and country and was used to identify the clinical specialty and country of origin.

Statistical Methods. Data were analyzed with linear regression curve estimation. Statistical analysis was performed by using $\mathrm{R}$ software (Windows version 2.6.2, R Foundation for Statistical Computing, Vienna, Austria).

\section{Results}

A total of 19,003 brain tumor journal articles were identified worldwide for the study period (1996-2007 inclusive) by using the major MeSH keywords of "brain neoplasm." In addition to brain neoplasms, the articles also covered a total of 1880 unique subjects that were designated as major topics by the MeSH of PubMed. The most common additional topics were glioma (4407), glioblastoma (2357), astrocytoma (1787), and MR imaging (1342). Of the total brain tumor articles, $6292(33.1 \%)$ originated in the United States.

The United States was the dominant producer of brain tumor research, in terms of number of articles published during the study period (6292), followed by Japan (2270), Germany (1773), and France (847, Fig 1). Authors in the United States, the Netherlands, and Switzerland published articles in journals with high mean impact factors (Fig 1). The output from the United States increased at a rate of 11.1 articles per year during the study period, whereas other major contributing countries showed little change (Fig 2).

For brain tumor research, neurosurgery departments produced the most articles (4871), followed by pathology (1783) and radiology (1637) (Fig 3). Articles from neurosurgery and radiology departments were published in journals with lower mean journal impact factors than several other specialties, including pathology, neurology, and hematology-oncology. The

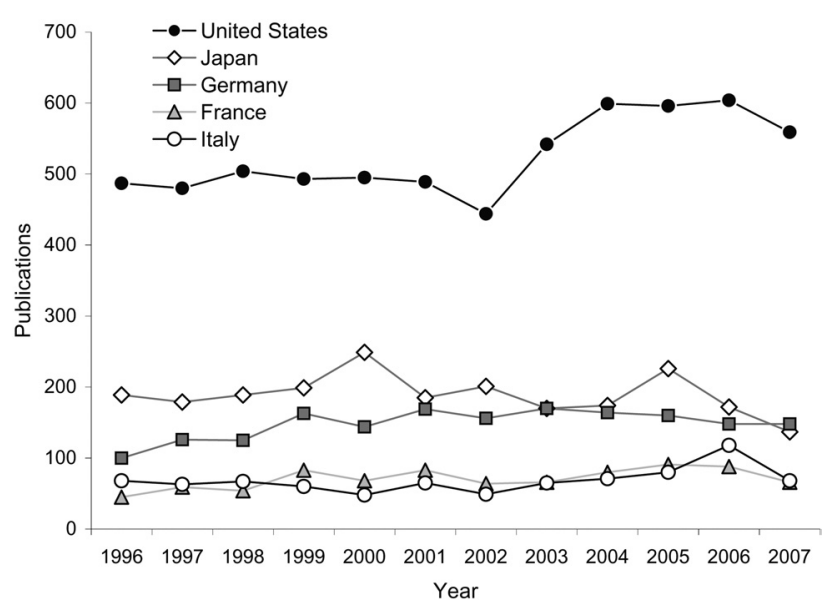

Fig 2. Number of publications by year for the top 5 countries by publication output. The trend for the United States is upwards. Linear regression curve fitting demonstrates growth in yearly US output at the rate of 11.1 articles per year $(P=.006)$.

journal impact factor for articles from radiology departments averaged 2.2, compared with 3.4 for hematology-oncology, but trended up during the 12-year study period.

The only radiology journal in the top 10 ranked journals, according to the number of articles published, was $A J N R$ (ranked number 8). AJNR published 281 articles on brain neoplasms during the study period, compared with 1179 publications for the Journal of Neuro-oncology, which published the most. As a whole, radiology journals published the third highest number of neuro-oncology articles, behind pathology- and neurosurgery-focused journals, mirroring the ranking for author specialty.

The proportion of brain tumor articles funded by NIH is substantial and growing. For articles originating in the United States, 2037 (32.4\%) acknowledged NIH grant support, averaged during the entire study period. The proportion of NIHfunded articles rose during the study period from $28.1 \%$ in 1996 to $36.7 \%$ in 2007. As expected, clinical trials (46.5\%) and general research articles $(44.2 \%)$ were funded by the NIH more often than other article types such as reviews (16.1\%) and case reports (5.2\%). The proportion of NIH-funded articles as well as general research and clinical trial subtypes grew in the study period (Fig 4). The proportion of NIH-funded brain tumor articles from radiology was $32.3 \%$ versus $32.4 \%$ for neurosurgery, 33.5\% for pathology, 32.9\% for neurology, and $40.1 \%$ for hematology-oncology (Fig 5). The percentage of brain tumor articles published by radiology departments and funded by the NIH grew at an average rate of 2.5 percentage points per year, faster than that in other specialties (Fig 6).

\section{Discussion}

The level of NIH funding is the single greatest predictor of research output among US radiology departments, and approximately $86 \%$ of the number of publications can be predicted by NIH funding levels alone. ${ }^{4}$ NIH funding levels are scheduled to get a one-time boost of $\$ 8.2$ billion in extramural funding associated with the American Recovery and Reinvestment Act of 2009. Therefore, we quantified the world output of published brain tumor research and studied the geographic origin, scientific methodology, publication trends, and associated NIH funding during a recent 12 -year period. 


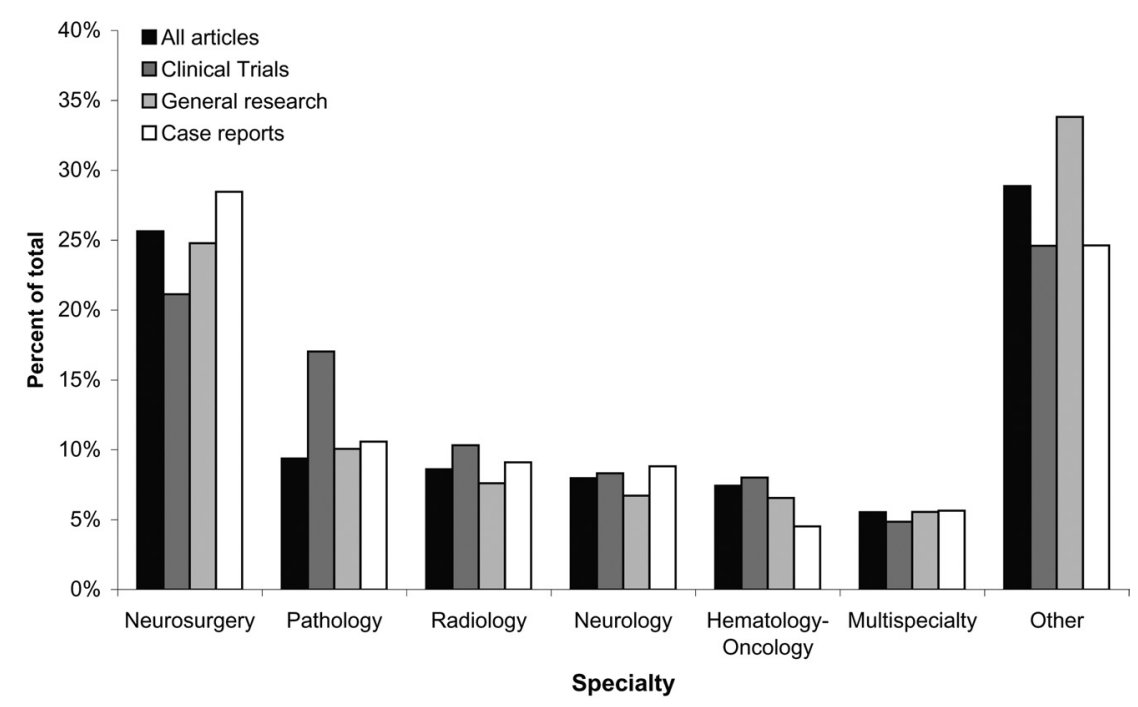

Fig 3. Specialty contributions to total brain tumor articles. Neurosurgery departments produced the most publications during the study period.

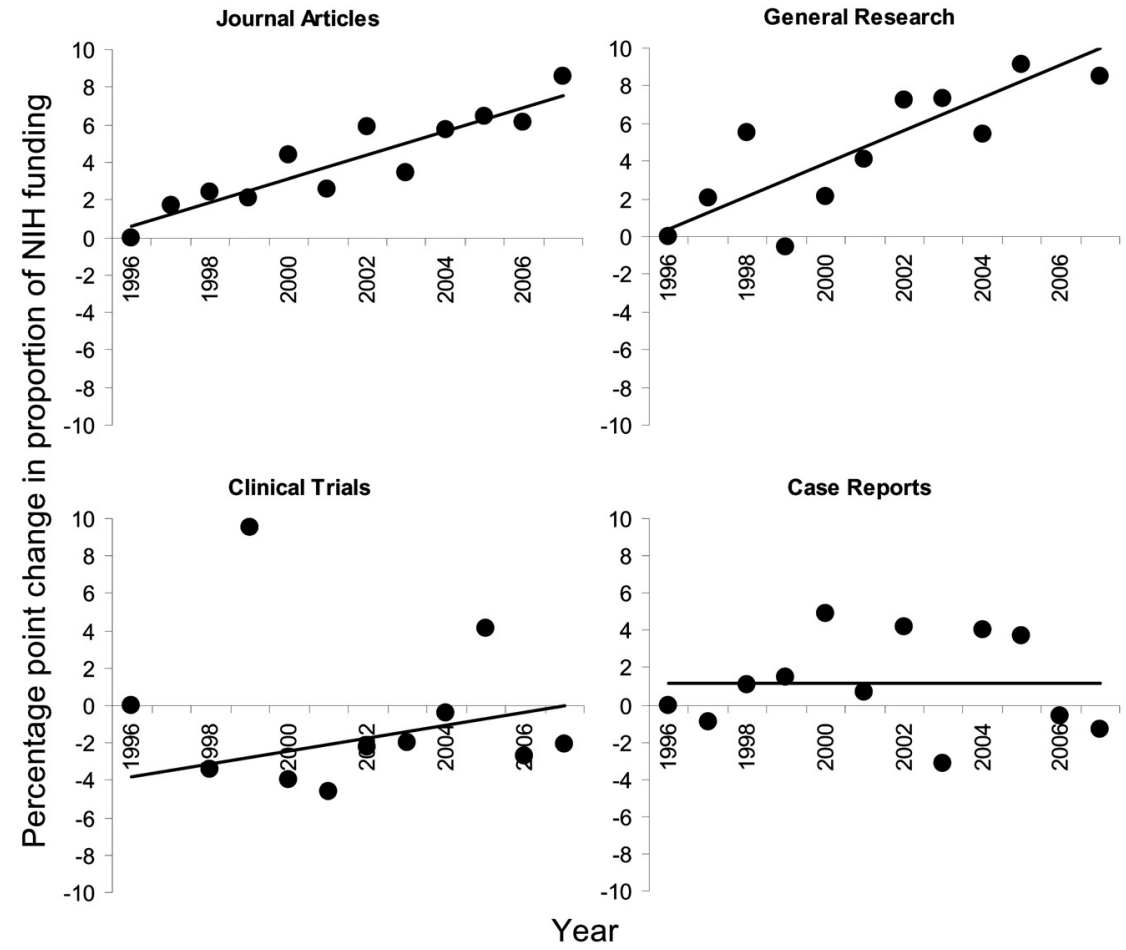

Fig 4. Growth in NIH funding of US brain tumor publications by article type. Lines represent the predicted linear growth trend. A data point in the clinical trials graph (year 1997, -15.05) is not depicted. The proportion of brain tumor research funded by the NIH is substantial and growing for general research articles, clinical trials, and overall journal articles.

The overall NIH yearly budget for the study period increased from $\$ 11.9$ billion to $\$ 29$ billion (a $143 \%$ increase). Funding for brain cancer has also increased. For the period 2003-2009, years for which data specific for brain cancer funding are available (http://report.nih.gov/rcdc/categories/, updated May 7, 2009), NIH funding for brain tumor research increased from $\$ 164$ million to $\$ 200$ million per year. Most of this research is supported by the National Cancer Institute (NCI Office of Budget and Finance. http://obf.cancer.gov/).

During our 12-year study period, the United States was the dominant producer of brain tumor research, resulting in nearly 3 times as many articles published on brain neoplasms compared with the next most prolific country (Japan). The proportion of US research funded by the NIH rose by $>10 \%$ during the study period. The mean impact factor for journals in which these articles were published also was substantially higher than that in most other countries. The years 2002-2004 showed the greatest change in US output, which rose approximately 20\% during those 3 years alone. NCI funding of brain tumor research also increased rapidly for the years before and during this time period (from $\$ 54$ million in 1998 to $\$ 132$ million in 2004, an approximately 2.4 -fold increase) before leveling off. In 2005, there was a substantial cut in NIH brain tumor research funds from $\$ 187$ million to $\$ 157$ million, and the number of research publications from the United States was stable from 2004 to 2006 before dropping slightly in 2007. 


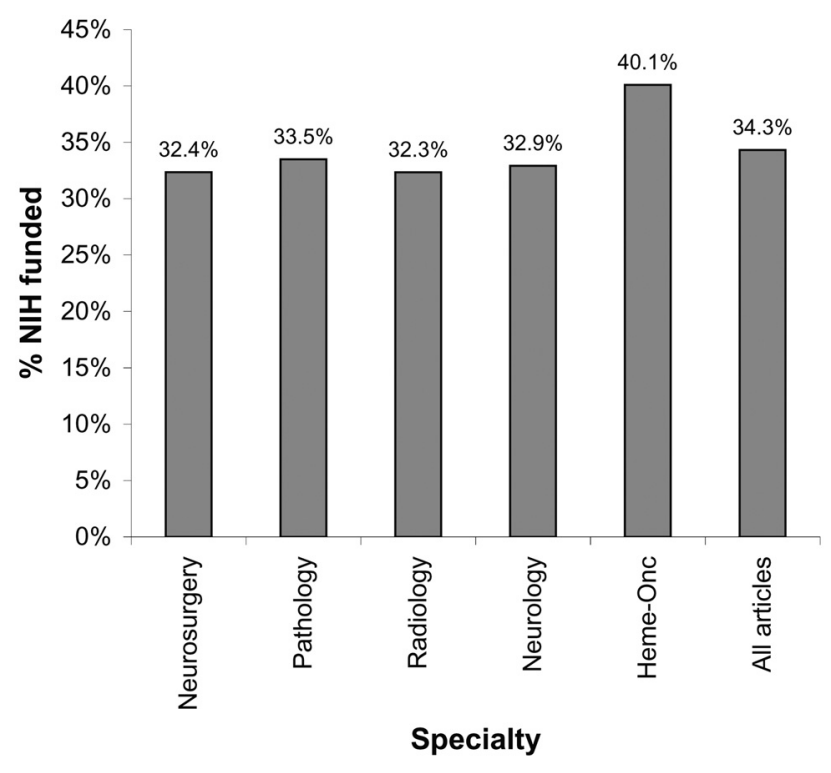

Fig 5. The proportion of US brain tumor research funded by the NIH is similar across specialties, excepting hematology-oncology, which shows a slightly higher percentage of $\mathrm{NIH}$-funded articles.

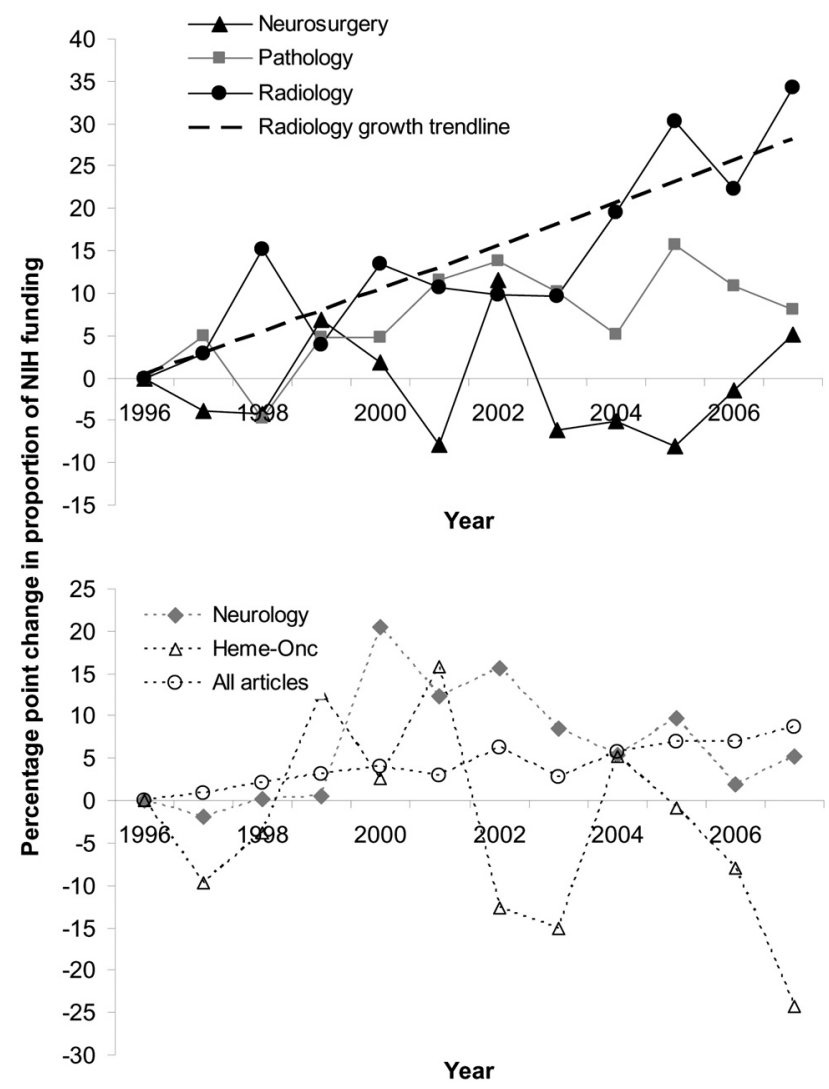

Fig 6. Change in the proportion of $\mathrm{NIH}$-funded articles by clinical specialty. The $\mathrm{y}$-axis is the percentage point change since 1996. The proportion of publications from radiology departments funded by the $\mathrm{NIH}$ increased significantly, as demonstrated by the linear regression curve fit.

Clearly, there is not a one-to-one correspondence between funding levels and publications on a yearly basis, and our data do not prove a causal relationship between funding and research output. However, data are not inconsistent with the hypothesis that increased funding has a positive impact on research publication levels. If that is the case, the data suggest that there may be a time lag of several years between increased spending on research and increased publication rates.

There are several interesting differences in brain tumor research funding between the United States and Europe. For instance in 2005, approximately $54 \%$ of brain research was government funded in the United States, (industry is the other major source of funding) compared with 16\% in Europe. ${ }^{11,12}$ Brain research funding per capita is approximately 5 times higher in the United States than in Europe. ${ }^{13}$ European government funding of brain tumor research was approximately $12 \%$ of NIH funding of brain tumor research in 2005 . $^{11,12}$ Although Europe has more than twice the population of the United States, the number of European publications (5941) was roughly equivalent to that of the United States (6292). Thus the United States had substantially more government funding of brain tumor research than Europe, and the per capita publication output was also higher, again supporting a positive relationship between government funding levels and research output.

For our data, approximately $10 \%$ of brain tumor research was published by radiology departments. NIH funding levels for brain tumor articles were roughly the same for radiology as for other specialties, but this proportion was higher than NIHfunding levels for radiology research as a whole. Thus, we found that approximately $30 \%$ of brain tumor publications from radiology departments were $\mathrm{NIH}$-funded, compared with $<20 \%$ for radiology articles in general. ${ }^{10}$ The impact factor for journals publishing radiology brain tumor articles is lower than that for several other specialties (pathology, neurology, and hematology-oncology), but similar to that for neurosurgery. However, this observation has to be viewed with some caution because there are limitations in comparing impact factors across specialties. For instance, it is known that there are a variety of characteristics that can cause a specialty to have a lower or higher impact factor even when the same or equivalent amount of research is being done. Factors include readership, citation half-life, the number of citations on average per paper, and stage in the development cycle (basic sciences tend to be cited more than clinical sciences, the former often being cutting edge and the latter being more application).

The impact factor for brain tumor articles with the first author from a radiology department trended upward during the study period. The only radiology journal in the top 10 ranked journals, according to the number of articles published, was AJNR (ranked number 8). As a whole, radiology journals published the third highest number of neuro-oncologic articles, behind pathology- and neurosurgery-focused journals.

Our analysis has several limitations. For instance, this research was based on data collected from the PubMed data base of the National Library of Medicine. While PubMed is generally considered to be comprehensive and accurate, errors in PubMed affecting article categorization of methodologies could bias the results. Additionally, if there are systematic differences in reporting of NIH funding that vary by location, then the results could be biased. Another limitation is that not all journals indexed by PubMed have been assigned impact factors by Thompson Reuters. Articles in these journals were 
excluded in the calculation of the journal impact factor. Because journals not indexed tend to be more obscure, they would likely have lower impact factors. Therefore, this may result in some positive bias in the mean journal impact factor. Finally, the methodology uses the first author's affiliation to assign the clinical specialty. In instances in which articles were collaborative efforts between multiple specialties, specialties of non-first authors would not receive credit, potentially leading to undercounting if authors from these specialties are systematically not first authors. This may be the case with certain authors with specialized skills such as statisticians. Conversely however, giving all authors equal credit could lead to overcounting of non-first authors. For example, should 10 clinical trials on surgery with a statistician as fourth author count as 10 primary statistics papers? Several studies have determined that the first author does the greatest volume of work and is most deserving of credit. ${ }^{14,15}$ We thought that using the first author to assign the clinical specialty was the best, if imperfect, strategy.

Our analysis suggests a growing importance of NIH funding levels for brain tumor research. Because radiology departments contribute substantially to this area of investigation, we suggest that radiology departments, especially those conducting brain tumor research, could be positively impacted by budgeted increases in NIH funding levels. Given the one-time boost of $\$ 8.2$ billion in NIH extramural research funding during the next 2 years, it will be of interest to see how US radiology research publication levels are impacted in the near future.

\section{Conclusions}

Radiology departments contribute substantially to NIHfunded brain tumor research. Brain tumor research is funded by the NIH at higher levels than radiology research as a whole and may be positively impacted by impending increases in NIH funding levels.

\section{References}

1. American Cancer Society Facts \& Figures 2009. Atlanta: American Cancer Society Available at: http://www.cancer.org/downloads/STT/500809web.pdf. Accessed October 13, 2009

2. McLendon RE, Halperin EC. Is the long-term survival of patients with intracranial glioblastoma multiforme overstated? Cancer 2003;98:1745-48

3. Jemal A, Siegel R, Ward E, et al. Cancer statistics, 2008. CA Cancer J Clin 2008; 58:71-96. Epub 2008 Feb 20

4. Itagaki MW, Pile-Spellman J. Factors associated with academic radiology research productivity. Radiology 2005;237:774-80

5. McAllister PR, Narin F. Characterization of the research papers of U. S. medical schools. J Am Soc Inf Sci 1983;34:123-31

6. Jonisch AI, Kilgermna S, Nagy E, et al. What characterizes radiology departments that secure large amounts of external funding for research? Acad Radiol 2006;13:1513-16

7. Maquire MA, JC Gore. The current state of NIH funding of research in diagnostic radiology at U.S. medical schools. J Am Coll Radiol 2005;2:436-43

8. Steinbrook R. Health care and the American Recovery and Reinvestment Act. N Engl J Med 2009;360:1057-60

9. Steinbrook R. The NIH stimulus: the recovery act and biomedical research. N Engl J Med 2009;360:1479-81

10. Itagaki MW. Impact of the National Institutes of Health on radiology research. Radiology 2008;247:213-19

11. Sobocki P, Lekander I, Berwick $S$, et al. Resource allocation to brain research in Europe (RABRE). Eur J Neurosci 2006;24:2691-93

12. Sobocki $P$, Olesen J, Jönsson B. Brain research has high returns but Europe is lagging behind. Eur J Neurol 2007;14:708-10

13. Jäger M, Lekander I, Sobocki P, et al. Resource allocation to brain research in Switzerland. Swiss Med Wkly 2008;138:335-39

14. Slone RM. Coauthors' contributions to major papers published in the AJR: frequency of undeserved coauthorship. AJR Am J Roentgenol 1996;167:571-79

15. Chew FS. The scientific literature in diagnostic radiology for American readers: a survey and analysis of journals, papers, and authors. AJR Am J Roentgenol 1986;147:1045-55 